strickungen in den Blick zu nehmen (Schulz, 1973, S. 758). Auf sozial unverantwortliches Verhalten soll eher pragmatisch, von einem »sozial-rechtlichen Schuldbegriff « (Baumann, 1969, S. 12) ausgehend, so reagiert werden, dass über Lernvorgänge die Einübung in sozial verträgliches Handeln ohne Straftaten möglich wird. Unser Wissen über die Ursachen und den Verlauf abweichenden Verhaltens zeigt, dass dieses Programm in vielen Fällen erfolgreich ist und zum gesellschaftlichen Frieden beiträgt. Viele Bedienstete in den Anstalten setzen diese mühselige Arbeit auch unter widrigen Umständen fort, z.T. allein gelassen von der politischen Spitze. $\mathrm{Zu}$ den herkömmlichen, in den Ländern über viele Jahre hinweg verschuldeten Mängeln treten nun intensive restaurative Tendenzen. Sie behindern die Orientierung am einzelnen Menschen, weil sie ressortegoistisch allein auf eine risikolose VerbüBung setzen, sich um das Danach nicht scheren und den Täter erneut auf Eigenschaften reduzieren, die geeignet sind, eigene Einsperrungsund Strafbedürfnisse zu rechtfertigen (s. dazu Böllinger, 2004).

In einer solchen Situation ragt das StVollzG wie ein Relikt aus besonneneren Tagen in die Gegenwart. Es gibt zu dessen Zielen, Leitsätzen und zu einigen zentralen Einzelbestimmungen keine vernünftige Alternative. Sein Geist und seine Inhalte würden durch die Aufgabe der zentralen Zuständigkeit noch mehr verraten. Das Hamburger Beispiel zeigt dies exemplarisch besonders deutlich: Wer, wie Senator Kusch, den offenen Vollzug mehr als halbiert, Lockerungen annähernd halbiert, Haftbedingungen auch sonst weiter verschärft und eine funktionierende Sozialtherapie durch eine unsinnige und teure Verlagerung zerstört (um hier nur die wichtigsten Punkte $\mathrm{zu}$ nennen) und bei allem glauben macht, es werde dadurch mehr Sicherheit für Hamburg hergestellt, der verschleudert vollzugspolitisches Kapital und verschärft die Probleme (Rehn, 2003). Das haben dem Senator, dem Ersten Bürgermeister und den Bürgerschaftsfraktionen viele Wissenschaftler und Praktiker schriftlich gegeben. Was immer daraus entstehen mag: Das Strafvollzugsgesetz, das so sehr in das Leben von Menschen in extrem abhängiger Position eingreift, darf nicht in die Hände der Länder fallen und dort auf die schiefe Bahn wech- selnder und sachferner Interessen geraten. Politik ist manchmal nur halbstark, dort am meisten, wo sie besonders stark auftritt.

\section{Literatur:}

Baumann, J. (1969): Schuld und Sühne als Grundproblem heutiger Strafrechtspflege. In: J. Baumann (Hrsg.) (1969): Misslingt die Strafrechtsreform? Neuwied und Berlin: Luchterhand

Böllinger, L. (2004): Schuld-Strafe-Macht: Eine psychosoziale Rekonstruktion. In: G. Rehn, R. Nanninga \& A. Thiel (Hrsg.): Freiheit und Unfreiheit. Arbeit mit Straftätern innerhalb und außerhalb des Justizvollzuges. Herbolzheim: Centaurus, S. 53-77

Callies, R.-P. (2001): Die Strafzwecke und ihre Funktion. Straftheorie oder dialogische Strafrechtstheorie als Bezugsrahmen. In: G. Britz, H. Jung, H. Koriath \& E. Müller (Hrsg.): Grundfragen staatlichen Strafens. Festschrift für Heinz Müller-Dietz zum 70. Geburtstag, München: C. H. Beck, 99-118

Dünkel, F. (2003): Sicherheit als Vollzugsziel - Die Wende im Strafvollzug in Zeiten des Wahlkampfes: eine Initiative aus Hessen. In: Neue Kriminalpolitik 1/2003
Dünkel, F. (2004): Riskante Freiheiten? - Vollzugslockerungen zwischen Resozialisierung und Sicherheitsrisiko. In: G. Rehn, R. Nanninga \& A. Thie (Hrsg.): Freiheit und Unfreiheit. Arbeit mit Straftätern innerhalb und außerhalb des Justizvollzuges. Herbolzheim: Centaurus, S. $104-134$

Einsele, H. (1970). Referat. In: Ständige Deputation des Deutschen Juristentages (Hrsg.). Mit welchem Hauptinhalt empfiehlt es sich, ein Strafvollzugsgesetz zu erlassen? Verhandlungen des 48 Deutschen Juristentages 1070 in Mainz. München: Beck, S. 32-51.

Feest, J. \& W. Lesting (2004): Der Angriff auf die Lockerungen. Daten und Überlegungen zur Lockerungspolitik der Länder. In ZfStrVo (in Vorbereitung)

Jehle, J.-M., W. Heinz \& P. Sutterer (2003): Legalbewährung nach strafrechtlichen Sanktionen, Berlin: Bundesministerium der Justiz

Kaiser, G \& H. Schöch (2002): Strafvollzug. Eine Einführung in die Grundlagen. 5 Aufl. Karlsruhe: C.F.Müller

Kerner, H.-J. (2004): Freiheit und Unfreiheit. Zum Verlauf der Karrieren von Straftätern. In: G. Rehn, R. Nanninga $\&$ A. Thiel (Hrsg.): Freiheit und Unfreiheit. Arbeit mit Straftätern innerhalb und außerhalb des Justizvollzuges. Herbolzheim: Centaurus, $3-52$
Rehn, G. (2003): Ist eine rationale Strafvollzugspolitik heute noch möglich? Strafvollzug am Scheideweg. In: ZfStrVo, S. 70 - 76

Rehn, G. (2004): Reform und Gegenreform. Bemerkungen zur Situation und zu den Perspektiven des Strafvollzuges. In: G. Rehn, R. Nanninga \& A. Thiel (Hrsg.): Freiheit und Unfreiheit. Arbeit mit Straftätern innerhalb und außerhalb des Justizvollzuges. Herbolzheim: Centaurus, 523 -537

Schöch, H. (1996): Die Rechtswirklichkeit und präventive Effizienz strafrechtlicher Sanktionen. In: J.M. Jehle (Hrsg.): Kriminalprävention und Strafjustiz. Wiesbaden: Selbstverlag Kriminologische Zentralstelle, Bd. 17, S. 291-326

Schulz, W. (1972): Philosophie in der veränderten Welt. Pfullingen: Günther Neske

Stratenwerth, G. (1969): Schuld und Rechtfertigung. In: J. Baumann (Hrsg.) (1969): Misslingt die Strafrechtsreform? Neuwied und Berlin: Luchterhand

Dr. Gerhard Rehn, 1984 bis 1994 Leiter der Sozialtherapeutischen Anstalt Hamburg-Altengamme und bis 2000 Leiter der Abteilung Vollzugsgestaltung in der Justizbehörde Hamburg

\title{
Strafvollzug ist Ländersache!? Was trotz bundeseinheitlicher Gesetzgebungs- kompetenz des Bundes möglich ist
}

\section{- Dargestellt am Beispiel des offenen Vollzuges}

Frieder Dünkel

( ie negativen Folgen der von Gerhard Rehn (siehe nebenstehenden Bericht) thematisierten Verlagerung der Bundes- auf eine Landeskompetenz, wie sie die (insoweit glücklicherweise gescheiterte) sog. Föderalismuskommission vorgeschlagen hatte, können am nachfolgenden Beispiel des offenen Vollzugs eindrucksvoll belegt werden. Der offene Vollzug ist (ebenso wie andere Resozialisierungsmaßnahmen wie Vollzugslockerungen und Hafturlaub) zum Spielball restaurativer Vollzugspolitik geworden (vgl. schon Dünkel/Kunkat NK 2/1997). Das Strafvollzugsrecht betrifft einen Kernbereich staatlicher Tätigkeit, im Rahmen derer die intensivsten Eingriffe in die Rechte von verurteilten Bürgern stattfin- den. Der unsere Verfassung prägende Grundsatz, dass in den Ländern möglichst einheitliche Lebensverhältnisse herzustellen sind, muss im Strafvollzug in besonderem Maße Beachtung finden. Es war erklärtes Ziel der mehr als 100-jährigen Gesetzgebungsgeschichte bis zur Verabschiedung des Strafvollzugsgesetzes im Jahr 1976, der Auseinanderentwicklung der Lebensverhältnisse in den einzelnen Bundesländern entgegen $\mathrm{zu}$ wirken. Deshalb wurden $u$. a. zeitgleich mit dem StVollzG bundeseinheitliche Verwaltungsvorschriften erlassen.

Die nachfolgenden Daten verdeutlichen, dass schon der derzeitige Zustand des Strafvollzugs der Vorgabe einigermaßen vergleichbarer Le- bensverhältnisse und des unumstritten sinnvollen Ausbaus des offenen Vollzugs als besonders resozialisierungsfördernder Maßnahme immer weniger entsprochen wird. Allein seit 1996 hat sich der stichtagsbezogene Anteil von Gefangenen im offenen Vollzug in einigen Bundesländern drastisch verändert. Hamburg, ehemals liberale Hochburg eines resozialisierungs- und überleitungsorientierten Vollzugs, hat unter der konservativen Regierung und Justizsenator Kusch den Anteil von über $30 \%$ auf knapp 15\% mehr als halbiert. Hessen, ein Bundesland, das den offenen Vollzug als eines der ersten schon in den 1950er und 1960er Jahren modellhaft ausgebaut hatte, hat seine erfolgreiche Strategie (einschließlich der Direkteinweisung 
Abbildung 1: Anteil von Strafgefangenen im offenen Vollzug 1996 und 2004

(Erwachsenenstrafvollzug, Stichtage 30.6.1996 und 31.3.2004)*

\begin{tabular}{|l|c|c|}
\hline Bundesland & 1996 & 2004 \\
\hline Baden-Württemberg & $18,7 \%$ & $18,5 \%$ \\
\hline Bayern & $7,1 \%$ & $7,8 \%$ \\
\hline Berlin & $30,6 \%$ & $32,9 \%$ \\
\hline Brandenburg & $15,2 \%$ & $13,1 \%$ \\
\hline Bremen & $19,9 \%$ & $12,8 \%$ \\
\hline Hamburg & $31,3 \%$ & $14,6 \%$ \\
\hline Hessen & $27,3 \%$ & $10,1 \%$ \\
\hline Mecklenburg-Vorpommern & $12,5 \%$ & $13,4 \%$ \\
\hline Niedersachsen & $28,4 \%$ & $25,5 \%$ \\
\hline Nordrhein-Westfalen & $29,0 \%$ & $31,2 \%$ \\
\hline Rheinland-Pfalz & $16,3 \%$ & $13,4 \%$ \\
\hline Saarland & $21,5 \%$ & $21,0 \%$ \\
\hline Sachsen & $6,1 \%$ & $9,6 \%$ \\
\hline Sachsen-Anhalt & $3,0 \%$ & $6,4 \%$ \\
\hline Schleswig-Holstein & $13,1 \%$ & $9,5 \%$ \\
\hline Thüringen & $3,0 \%$ & $7,5 \%$ \\
\hline Alte Bundesländer insg. & $22,5 \%$ & $20,5 \%$ \\
\hline $\begin{array}{l}\text { Neue Bundesländer } \\
\text { insgesamt }\end{array}$ & $7,7 \%$ & $9,7 \%$ \\
\hline BRD insgesamt & $20,8 \%$ & $18,7 \%$ \\
\hline
\end{tabular}

* Quelle: Dünkel/Kunkat NK 2/1997, S. 26 (für 1996); für 2004: eigene Berechnungen nach www.destatis.de, Onlinepublikationen des Statistischen Bundesamts.

von nicht gefährlichen Gefangenen in den offenen Vollzug) unter der erklärten politischen Kehrtwende konservativer Regierungspolitik (vgl. Justizminister Wagner) aufgegeben (vgl., hierzu Dünkel/Drenkhahn NK 2/2001, S. 16 ff., 20) und den offenen Vollzug weitgehend zerschlagen: Noch 1996 waren $27 \%$ der Gefangenen im offenen Vollzug untergebracht, jetzt sind es noch 10\%. Damit liegen diese Bundesländer im Bereich des niedrigen »Ost-Niveaus" von Sachsen, Sachsen-Anhalt oder Thüringen und dem immer schon besonders restriktiven Bayern (7-8\%). ${ }^{1}$

Andere Bundesländer wie Berlin (2004: 33\% der Freiheitsstrafe verbüßenden Gefangenen im offenen Vollzug) oder Nordrhein-Westfalen (31\%) haben ihre bewährte Praxis beibehalten. Dies ersichtlich ohne negative Konsequenzen! Entweichungen und Straftaten während Lockerungsmaßnahmen im offenen Vollzug sind überall die Ausnahme. Die liberalere Praxis in Berlin und NRW bei einer vergleichbaren Insassenstruktur widerlegt das aus Hamburg und Hessen zu hörende Argument, dass die Insassen immer gefährlicher würden und damit un- desstrafvollzugsgesetze, sondern eine Qualitätssicherung bzgl. bewährter Resozialisierungskonzepte (wie des offenen Vollzugs) sowie das Festhalten am Prinzip des geringst möglichen Eingriffs in Grundrechte wie sie dem Geist des StVollzG entsprechen. Insoweit muss das Prinzip des Vorrangs des offenen Vollzugs erhalten bleiben (vgl. § 10 Abs. 1 und 2 StVollzG), auch wenn es vielleicht auf Dauer nur eine reale Utopie bleiben wird.

Prof. Dr. Frieder Dünkel lehrt Kriminologie und Strafrecht an der Universität Greifswald und ist Mitherausgeber dieser Zeitschrift

\section{Fußnote:}

1 Mit Bayern und anderen Ländern mit einem unterentwickelten offenen Vollzug nicht vergleichbar ist Schleswig-Holstein, das ebenfalls eine relativ geringe Quote von Gefangenen im offenen Vollzug aufweist (2004: knapp 10\%) Schleswig-Holstein hat die bundesweit niedrigste Inhaftierungsrate (vgl. Dünkel/Geng NK 2003, S. $146 \mathrm{ff}, 148: 59$ pro 100.000 gegenüber 99 in Bayern und mehr als 100 bzw. 110 in Sachsen und Sachsen-Anhalt) und daher tatsächlich einen geringeren Anteil von für den offenen Vollzug geeigneten Gefangenen.

12 Fragen an die Bundesjustizministerin zur geplanten Verlagerung der Kompetenzen zum Strafvollzugsrecht auf die Länder

1. Trifft es zu, dass der Vorschlag der Verlagerung der Zuständigkeit für den Strafvollzug auf die Länder vom Bundesjustizministerium eingebracht wurde?

Wenn ja, was waren die Gründe dafür?

2. Warum wird der partei- und länderübergreifende Konsens, der in den 1970er Jahren zum bundeseinheitlichen Strafvollzugsgesetz (und dazu ergänzend: bundeseinheitlichen Verwaltungsvorschriften zum StVollzG) geführt hat, durch diesen Vorschlag gefährdet?

3. Wird die Gefahr nicht gesehen, dass bundesweit einheitliche Regeln und Standards aufgegeben werden? Nahezu alle Experten erwarten einen »Wettbewerb der Schäbigkeit«, eine Absenkung der Standards und Ressourcen auf ein niedrigeres Niveau. (vgl. Stellungnahme der führenden deutschen Vollzugswissenschaftler, Strafrechtler und Kriminologen).

4. Warum wird der Anspruch bzgl. einer Rechtseinheit in den Bereichen des materiellen Strafrechts, des Strafverfahrensrechts und des Strafvollzugsrechts aufgegeben?

5. Wird die Gefahr nicht gesehen, dass auf Grund von spektakulären Einzelfällen der jeweilige Landesgesetzgeber vorschnell das Landesstrafvollzugsgesetz ändert (z. B. weniger Urlaub, Ausgang, Freigang, Überbetonung von Sicherheit)?

6. Was wird aus den von der Praxis dringend benötigten Entwürfen zum Jugendstrafvollzugsgesetz und zum Untersuchungshaftvollzugsgesetz? Werden Sie die Verabschiedung dieser Gesetze weiter mit aller Kraft vorantreiben?

7. Was wird aus dem Modell des deutschen Strafvollzugs, das zur Zeit in vielen (ost-) europäischen Ländern als beispielhaft gilt und »exportiert« wird?

8. Wie passt das Bestreben, innerhalb des Europarats bzw. der EU mittelfristig zu einheitlichen Strafvollzugsstandards zu kommen (vgl. das Problem von Überstellungen) zu der beabsichtigten neuen »Kleinstaaterei« in Deutschland?

9. Warum gab es zu diesem Thema keine bundesweite Fachdiskussion, keine Beteiligung von Wissenschaft, Praxis und von Fachorganisationen? Warum wurde die Justizministerkonferenz nicht beteiligt?

10. Soll nunmehr die Fachdiskussion nachgeholt werden?

11. Welche Länder haben sich für welche gegen eine Verlagerung der Bundeskompetenz ausgesprochen?

12. Werden noch Chancen gesehen, die Bundeskompetenz zu erhalten und werden Sie sich persönlich dafür einsetzen?

Für Rückfragen stehe ich jederzeit gerne zur Verfügung.

Mit freundlichen Grüßen

gez. Prof. Dr. Frieder Dünkel 